\title{
Commentary
}

\section{The evolving CRISPR technology}

\author{
Meng Yan, Jinsong $\mathrm{Li}^{\bowtie}$
}

State Key Laboratory of Cell Biology, Shanghai Key Laboratory of Molecular Andrology, CAS Center for Excellence in Molecular Cell Science, Shanghai Institute of Biochemistry and Cell Biology, Chinese Academy of Sciences, University of Chinese Academy of Sciences, Shanghai 200031, China

$\bowtie$ Correspondence: jsli@sibcb.ac.cn (J. Li)

Advances in genome editing technology have revolutionized basic and applied biology research in recent years, particularly due to the newly emerged CRISPR/Cas technique (Ren et al., 2017b). The classical CRISPR/Cas system was derived from a bacterial defense system, which consists of a single guide RNA (sgRNA) for precise targeting and one Cas protein for DNA binding and nuclease activity. Relying on high precision of CRISPR/Cas system, multiple functions have been exploited in addition to original nuclease activity, such as genome base editing, gene knock-down and activation, chromatin imaging systems etc. (Barrangou and Doudna, 2016; Wang and Qi, 2016). These extensions have demonstrated the flexibility of the CRISPR/Cas system as it has boosted basic biological, biomedical and agricultural research (Shen et al., 2017; Zhang et al., 2017). Here we summarize some key events in CRISPR/Cas system development, provide a glimpse of how this technology has transformed biological research, and help you to choose the right tool for your own research (Fig. 1).

CRISPR/Cas system was first introduced into human 293FT cells where it was shown to cleave target sites with high efficiency. The resulting double-stranded breaks (DSBs) in genomic DNA were partially repaired by the indel-forming nonhomologous end joining (NHEJ) pathway (Cong et al., 2013); therefore indels (insertions/deletions) and base mutations may present within the target site, which could deactivate the target gene and achieve in vivo gene knockout. The convenience and precision of this technique has

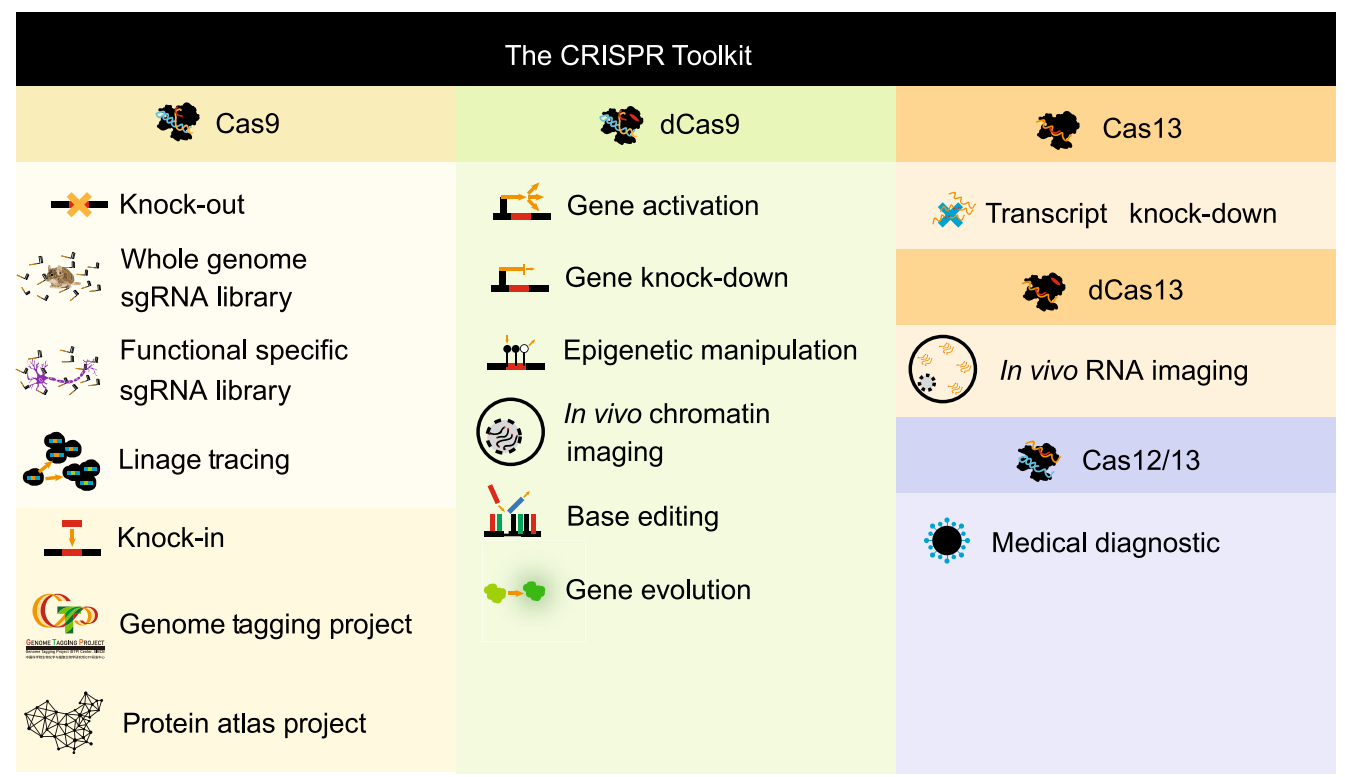

Figure 1. The CRISPR toolkit. 
facilitated its rapid adoption to the genome of a wide range of species, including human, monkey, mouse, rat, fly, zebrafish, Arabidopsis Thaliana and rice and so on. Because its targeting precision and efficiency have been well studied, targeting sites can be designed to minimize off-target binding and maximize on-target efficiency (Hsu et al., 2013). A recent research identified an advanced xCas9 variant with broad protospacer adjacent motif (PAM) compatibility and high DNA specificity, suggesting that higher editing efficiency, better PAM compatibility and DNA specificity can be achieved at the same time (Hu et al., 2018). In addition, based on the structure of Cas9, rationally engineered SpCas9-NG variant, also with an increased targeting range (higher activity towards NGD sites than NGC sites), promises a better efficiency over xCas 9 at NGD targeting sites and suggests a way to design Cas 9 for further optimization (Nishimasu et al., 2018).

Instead of a single sgRNA, a well-purposed sgRNA library could facilitate large-scale functional genomic studies (Shalem et al., 2014; Zhong et al., 2015), providing a competitive approach to screen key components in biological processes of interest. Tissue-specific, cancer-related, and whole genome-scale libraries have been built to study underlying biological pathways and drug resistance. The application of the low-cost, highly convenient and flexible CRISPR/Cas systems to medical research enables large-scale screenings that reduces total investment in research and development without compromising outcomes.

Homology-directed repair (HDR) pathway offers another option for cells to repair DSBs (instead of NHEJ). When a DNA template with a flanking homologous sequence is introduced, cells can repair DSBs with precise recombination at the DSBs site (CRISPR/Cas knock-in system). Utilizing the concept, we could repair the mutations that induce diesease, which propose a bright future in gene therapy ( $\mathrm{Wu}$ et al., 2013; Wu et al., 2015). In the meantime, to study pathology of human genetic diseases, especially polygenic diseases, we could mimic the disease mutations in model organisms easily by using this method, which, otherwise, might be extremely difficult to achieve by other methods. Take one step further, the concept could also be used to add a tag to a target, protein or RNA molecule. It has been shown that mouse models can be generated using "artificial spermatids" (Yang et al., 2012), by integrating with CRISPR/Cas knock-in system, the recently launched genome tagging project (GTP) will provide in vivo tagged mouse models at a researcher's request (Jiang et al., 2018). This project shows versatility and capability of CRISPR systems and brings in vivo research from the cellular level to the animal level. Based on GTP, a new project, termed protein atlas project (PAP), may be proposed to extend the horizon of GTP by focusing on the in vivo protein-protein interaction networks and spatiotemporal expression patterns on a large scale.

Over the past several years, great efforts have been made to innovate in the CRISPR/Cas system, including gene knock-down, activation, evolution, in vivo chromatin imaging, epigenetic modification, base editing and lineage tracing systems. These CRISPR/Cas subsidiaries have promoted multi-dimensional applications of original CRISPR/ Cas system in biological research, and its influences and improvements remain a hot topic.

Mutations generated by CRISPR/Cas system can be accumulated over cell divisions, and in combination with the advantages of next generation sequencing (NGS) technology, lineage relations between ancestral progenitors and descendants can be traced based on their mutation patterns. This approach allows us to record lineage information through developmental processes, and drew a lineage tree based on the information, which provides a good intuition of where particular cells are originated from and where they go (McKenna et al., 2016): it offers a roadmap of cell development on a specific time scale.

Gene knock-down and activation systems employ the nuclease-dead Cas enzyme (dCas9), the endonuclease activity of which has been destroyed due to point mutations in the $\mathrm{HNH}$ and RuvC domains, to manipulate gene expression in vivo (Gilbert et al., 2013; Maeder et al., 2013). The dCas9/sgRNA functions as a sniper, with versatile bullets such as transcriptional repressors or activators, aimed at the target site. If dCas9 carries repressors (KRAB), it will decrease target gene transcription; and if dCas9 carries activators (VP64 or VP128) or modified sgRNA with MS2binding site, it will increase expression of its target. Another way to achieve gene knock-down is by CRISPR/Cas13-an RNA-guided RNA-targeting system (Abudayyeh et al., 2017). CRISPR/Cas13 can cleave target transcripts in vivo with comparable efficiency and improved specificity using its own sgRNA for guidance. In addition, catalytically inactive Cas13a retains its RNA binding activity, and fused with florescent proteins, allows RNA movements in living cells to be tracked.

Fused with florescent proteins, dCas 9 can be used to visualize the dynamics of coding and noncoding segments of DNA within the host genome (Chen et al., 2013). For telomeres (which contains large amount of self-repeated sequences), only one or a few sgRNAs are needed; for DNA segments without repeated sequences, more sgRNAs are required to visualize chromatin in vivo. Improved signal/ noise ratio by splitting fluorescent protein or using the SunTag system, makes this imaging system more adaptive and practicable (Tanenbaum et al., 2014). Overall, the convenience and accuracy of this method eclipse that of traditional fluorescence -in situ-hybridization (FISH) method.

Laboratory evolution systems have great potential to generate enhanced genes, such as brighter florescent proteins and higher efficiency Cas enzymes. By coupling a nicking variant of Cas9 (nCas9) with an error-prone DNA polymerase, researchers have acquired EvolvR-an ideal targeted mutagenesis tool (Halperin et al., 2018), which allows to continuously mutate a target gene with a wide editing window. In addition, CRISPR-X and dCas9-AIDx systems have also been developed to perform gene 
evolution. Unlike EvolvR, these two utilize coupled cytidine deaminase to generate mutations within a limited editing window (Hess et al., 2016; Ma et al., 2016).

Epigenetic editing has been established as an essential tool for gene regulation. dCas 9 fused with the core catalytic domain of the human acetyltransferase p300 or lysine demethylase 1 can increase or decrease histone $\mathrm{H} 3$ (H3K27) acetylation of the target site, resulting in expression alterations (Thakore et al., 2016). Also, Dnmt3a or Tet1 fused dCas9 can increase or decrease CpG methylation levels near the target genomic site, respectively (Liu et al., 2016). These epigenetic manipulations could be used to investigate relations between epigenetic marks and gene spatiotemporal expressions in a series of key development transitions.

Base editing (BE) systems have been developed to tackle the task of correcting single base mutations in a genome, due to avoiding the uncertainty of NHEJ and low efficiency of HDR. Thus, BE systems provide a directional and highly efficient approach to correct disease-associated single nucleotide polymorphisms (SNPs). Currently there are two kinds of base editing systems: ABE for A-T-to-G.C editing and BE for C.G-to-T.A editing (Gaudelli et al., 2017; Kim et al., 2017; Ren et al., 2017a; Xue et al., 2018). The ABE system consists of an RNA adenosine deaminase fused nCas9 and a targeting sgRNA, and can introduce base switching more efficiently with a low rate of indel occurrence. The BE system uses cytidine deaminase fused nCas9 to achieve C.G-to-T.A editing, with higher efficiency but at the possible cost of causing genomic-scale mutations. The power of such tools has been harnessed to study gene functional regulation (including gain-of-function, increased or decreased gene activity) by inducing point mutations on a whole gene level ( $\mathrm{Li}$ et al., 2018). Future improvements to fine-tune the efficiency and off-target editing will be crucial for clinical applications of these tools (Koblan et al., 2018; Zuo et al., 2019).

Perhaps the most extraordinary aspect of the CRISPR/ Cas system is how it can transform medical research (Men et al., 2017; Zhang et al., 2017). In addition to the previously described genomic manipulation aspects, a new approach using Cas12a has emerged to detect infectious diseases (Chen et al., 2018). After the Cas12a-crRNA complex binds and cleaves target dsDNA specifically, its ability to cleave ssDNA is activated. When a cleavable fluorescent reportercoupled ssDNA is provided, this system can detect the existence of first dsDNA cleavage event, i.e. the existence of target disease-related dsDNA. Meanwhile, various methods based on Cas13 have been developed with much higher accuracy and simpler diagnostic procedures (SHERLOCK, SHERLOCKv2, and HUDSON combined SHERLOCK for example) (Gootenberg et al., 2018).
Indeed, a tool as powerful, versatile and convenient as the CRISPR/Cas system is a gift to biological research. CRISPR/Cas system and its derived systems give us new ways to study the complexity of life (Fig. 1). Future studies will focus on improving its specificity and efficiency, minimizing its off-target side effects, and also developing new derivations and applications.

\section{ACKNOWLEDGEMENTS}

This work was supported by Genome Tagging Project and grants from the Chinese Academy of Sciences (XDB19010204), Shanghai Municipal Commission for Science and Technology (17411954900, 17JC1400900, 17JC1420102, 16JC420500), the National Natural Science Foundation of China (31530048, 81672117, 31730062 and 31821004).

\section{COMPLIANCE WITH ETHICS GUIDELINES}

Meng Yan and Jinsong Li declare that they have no conflict of interest. This article does not contain any studies with human or animal subjects performed by the any of the authors.

\section{OPEN ACCESS}

This article is distributed under the terms of the Creative Commons Attribution 4.0 International License (http://creativecommons.org/ licenses/by/4.0/), which permits unrestricted use, distribution, and reproduction in any medium, provided you give appropriate credit to the original author(s) and the source, provide a link to the Creative Commons license, and indicate if changes were made.

\section{REFERENCES}

Abudayyeh OO, Gootenberg JS, Essletzbichler P, Han S, Joung J, Belanto JJ, Verdine V, Cox DBT, Kellner MJ, Regev A et al (2017) RNA targeting with CRISPR-Cas13. Nature 550:280-284

Barrangou R, Doudna JA (2016) Applications of CRISPR technologies in research and beyond. Nat Biotechnol 34:933-941

Chen BH, Gilbert LA, Cimini BA, Schnitzbauer J, Zhang W, Li GW, Park J, Blackburn EH, Weissman JS, Qi LS et al (2013) Dynamic imaging of genomic loci in living human cells by an optimized CRISPR/Cas system. Cell 155:1479-1491

Chen JS, Ma EB, Harrington LB, Da Costa M, Tian XR, Palefsky JM, Doudna JA (2018) CRISPR-Cas12a target binding unleashes indiscriminate single-stranded DNase activity. Science 360:436439

Cong L, Ran FA, Cox D, Lin SL, Barretto R, Habib N, Hsu PD, Wu XB, Jiang WY, Marraffini LA et al (2013) Multiplex genome engineering using CRISPR/Cas systems. Science 339:819-823

Gaudelli NM, Komor AC, Rees HA, Packer MS, Badran AH, Bryson DI, Liu DR (2017) Programmable base editing of A.T to G.C in genomic DNA without DNA cleavage. Nature 551:464-471 
Gilbert LA, Larson MH, Morsut L, Liu ZR, Brar GA, Torres SE, SternGinossar N, Brandman O, Whitehead EH, Doudna JA et al (2013) CRISPR-mediated modular RNA-guided regulation of transcription in eukaryotes. Cell 154:442-451

Gootenberg JS, Abudayyeh OO, Kellner MJ, Joung J, Collins JJ, Zhang F (2018) Multiplexed and portable nucleic acid detection platform with Cas13, Cas12a, and Csm6. Science 360:439444

Halperin SO, Tou CJ, Wong EB, Modavi C, Schaffer DV, Dueber JE (2018) CRISPR-guided DNA polymerases enable diversification of all nucleotides in a tunable window. Nature 560:248-252

Hess GT, Fresard L, Han K, Lee CH, Li A, Cimprich KA, Montgomery SB, Bassik MC (2016) Directed evolution using dCas9-targeted somatic hypermutation in mammalian cells. Nat Methods 13:1036-1042

Hsu PD, Scott DA, Weinstein JA, Ran FA, Konermann S, Agarwala V, Li YQ, Fine EJ, Wu XB, Shalem O et al (2013) DNA targeting specificity of RNA-guided Cas9 nucleases. Nat Biotechnol 31:827-832

Hu JH, Miller SM, Geurts MH, Tang WX, Chen LW, Sun N, Zeina CM, Gao X, Rees HA, Lin Z et al (2018) Evolved Cas9 variants with broad PAM compatibility and high DNA specificity. Nature 556:57-63

Jiang J, Yan M, Li D, Li J (2018) Genome tagging project: tag every protein in mice through 'artificial spermatids'. Natl Sci Rev 6:394-396

Kim YB, Komor AC, Levy JM, Packer MS, Zhao KT, Liu DR (2017) Increasing the genome-targeting scope and precision of base editing with engineered Cas9-cytidine deaminase fusions. Nat Biotechnol 35:371-376

Koblan LW, Doman JL, Wilson C, Levy JM, Tay T, Newby GA, Maianti JP, Raguram A, Liu DR (2018) Improving cytidine and adenine base editors by expression optimization and ancestral reconstruction. Nat Biotechnol 36:843-846

Li Q, Li YJ, Yang SM, Huang S, Yan M, Ding YF, Tang W, Lou XW, Yin Q, Sun ZF et al (2018) CRISPR-Cas9-mediated base-editing screening in mice identifies DND1 amino acids that are critical for primordial germ cell development. Nat Cell Biol 20:1315-1325

Liu XS, Wu H, Ji X, Stelzer Y, Wu XB, Czauderna S, Shu J, Dadon D, Young RA, Jaenisch R (2016) Editing DNA methylation in the mammalian genome. Cell 167:233-247

Ma YQ, Zhang JY, Yin WJ, Zhang ZC, Song Y, Chang X (2016) Targeted AID-mediated mutagenesis (TAM) enables efficient genomic diversification in mammalian cells. Nat Methods 13:1029-1035

Maeder ML, Linder SJ, Cascio VM, Fu YF, Ho QH, Joung JK (2013) CRISPR RNA-guided activation of endogenous human genes. Nat Methods 10:977-979

McKenna A, Findlay GM, Gagnon JA, Horwitz MS, Schier AF, Shendure J (2016) Whole-organism lineage tracing by combinatorial and cumulative genome editing. Science 353:aaf7907

Men K, Duan XM, He ZY, Yang Y, Yao SH, Wei YQ (2017) CRISPR/ Cas9-mediated correction of human genetic disease. Sci China Life Sci 60:447-457
Nishimasu H, Shi X, Ishiguro S, Gao LY, Hirano S, Okazaki S, Noda T, Abudayyeh OO, Gootenberg JS, Mori H et al (2018) Engineered CRISPR-Cas9 nuclease with expanded targeting space. Science 361:1259-1262

Ren B, Yan F, Kuang YJ, Li N, Zhang DW, Lin HH, Zhou HB (2017a) A CRISPR/Cas9 toolkit for efficient targeted base editing to induce genetic variations in rice. Sci China Life Sci 60:516-519

Ren XJ, Holsteens K, Li HY, Sun J, Zhang YF, Liu LP, Liu QF, Ni JQ (2017b) Genome editing in Drosophila melanogaster: from basic genome engineering to the multipurpose CRISPR-Cas9 system. Sci China Life Sci 60:476-489

Shalem O, Sanjana NE, Hartenian E, Shi X, Scott DA, Mikkelsen TS, Heckl D, Ebert BL, Root DE, Doench JG et al (2014) Genomescale CRISPR-Cas9 knockout screening in human cells. Science 343:84-87

Shen L, Hua YF, Fu YP, Li J, Liu Q, Jiao XZ, Xin GW, Wang JJ, Wang XC, Yan CJ et al (2017) Rapid generation of genetic diversity by multiplex CRISPR/Cas9 genome editing in rice. Sci China Life Sci 60:506-515

Tanenbaum ME, Gilbert LA, Qi LS, Weissman JS, Vale RD (2014) A protein-tagging system for signal amplification in gene expression and fluorescence imaging. Cell 159:635-646

Thakore PI, Black JB, Hilton IB, Gersbach CA (2016) Editing the epigenome: technologies for programmable transcription and epigenetic modulation. Nat Methods 13:127-137

Wang FY, Qi LS (2016) Applications of CRISPR genome engineering in cell biology. Trends Cell Biol 26:875-888

Wu YX, Liang D, Wang YH, Bai MZ, Tang W, Bao SM, Yan ZQ, Li DS, Li JS (2013) Correction of a genetic disease in mouse via use of CRISPR-Cas9. Cell Stem Cell 13:659-662

Wu YX, Zhou H, Fan XY, Zhang Y, Zhang M, Wang YH, Xie ZF, Bai $M Z$, Yin $Q$, Liang D et al (2015) Correction of a genetic disease by CRISPR-Cas9-mediated gene editing in mouse spermatogonial stem cells. Cell Res 25:67-79

Xue CX, Zhang HW, Lin QP, Fan R, Gao CX (2018) Manipulating mRNA splicing by base editing in plants. Sci China Life Sci 61:1293-1300

Yang $\mathrm{H}$, Shi LY, Wang BA, Liang D, Zhong CQ, Liu W, Nie YZ, Liu J, Zhao J, Gao X et al (2012) Generation of genetically modified mice by oocyte injection of androgenetic haploid embryonic stem cells. Cell 149:605-617

Zhang XH, Wang LR, Liu MY, Li DL (2017) CRISPR/Cas9 system: a powerful technology for in vivo and ex vivo gene therapy. Sci China Life Sci 60:468-475

Zhong CQ, Yin Q, Xie ZF, Bai MZ, Dong R, Tang W, Xing YH, Zhang HL, Yang SM, Chen LL et al (2015) CRISPR-Cas9-mediated genetic screening in mice with haploid embryonic stem cells carrying a guide RNA library. Cell Stem Cell 17:221-232

Zuo E, Sun Y, Wei W, Yuan T, Ying W, Sun H, Yuan L, Steinmetz LM, Li Y, Yang H (2019) Cytosine base editor generates substantial off-target single-nucleotide variants in mouse embryos. Science 364:289-292 